Journal of Architectural Research and Development

Research Article

\title{
Research and Application of Medicinal Plants in Garden Landscapes
}

\author{
Zhongjian Fang \\ Guangzhou Shangjing Ecological Landscape Co., Guangzhou 510000, Guangdong Province, China
}

\begin{abstract}
Since early 2020, the concern about the frontliners who are fighting against the coronavirus disease 2019 (COVID-19) is worrying as this disease is surging up. Medicinal plants have contributed a role in this pandemic. The medicinal plant has regained its attention although it has the perception of being hay or woods. Traditional Chinese medicines are ubiquitous in which the source is from any plants. Especially, people are applying for traditional medicine as the element in the garden landscaping. With the increase in the people's attention to Chinese medicine, the medicinal plants-themed garden landscapes are constructed in more provinces and cities. Besides, the state also recommends the introduction of Chinese medicine in the school as part of science teaching materials.

Key words: Traditional Chinese medicine; Planning; Design
\end{abstract}

Publication date: September, 2020

Publication online: 30 September, 2020

* Corresponding author: Zhongjian Fang, shangjingshengtai@163.com

\section{Role of medicinal plants}

The main function of medicinal plants is to serve as materials for Chinese herbal medicine, with seed preservation and public education as the main role. Besides, medicinal plants are used for scientific research, the collection and preservation of medicinal plants, the exhibition in the unique landscape in the botanical gardens. At the same time, an increasing number of designers are applying medicinal plants to the community or park landscaping architecture ${ }^{[1]}$.

\section{Medicinal Plant Landscape Construction Features}

The medicinal plants are widely applied in landscape designing. Among many gardens, the medicinal plantstheme garden has gained a novel garden landscaping design. Not only meeting the scientific classifications and ecological requirements, but the medicinal plantsthemed landscape also pursuit of the beauty of the landscaping. This landscape architecture will lead people in achieving a pleasant mind and souls and providing health benefits. Medicinal plant landscape consists of the combination of "ecology", "natural science", "landscape science" and "horticulture and flowers" aspects in the establishment of Chinese herbal plants thematic garden planning and design, combined with plant ecology, drug taxonomy garden tours and other comprehensive elements as the consideration factors. After all, the designers should be familiar with the characteristics of medicinal plants in the application process as some medicinal plants are toxic ${ }^{[2-3]}$.

According to the ecological relationship of Chinese herbal plants, we create a space that is "open, closed, clustered and smooth" utilizing herbal plants, woody plants, vines, shrubs, groundcover plants and buildings as landscaping elements. The medicinal plants combined with different herbs, groundcover plants and other plants, forming a garden landscape with light and dark changes, put in and out of changes. Therefore, visitors can move to different sceneries while being clueless in path tracking. The combination of garden landscaping and traditional Chinese medicine provides health benefits such as achieving a healthy soul and mind. Building a "fitness path" in one of the garden paths is part of the cure, anti-ageing, invigorating Chinese herbal plants into a "fragrance treatment area" and "anti-ageing healthcare area". The addition of plants with volatile oil components in medicinal plant landscape increases the concentration of plant essence in the air, purifying the air through small waterfalls and sprays to increase the concentration of anions, creating 
a quiet, clean tourist destination and creating a place for medicinal plants to recuperate ${ }^{[4]}$.

The Chinese herbal plant cultures can be exhibited in two functional areas: the live plant display of Chinese herbs and the science exhibition of Chinese herbal culture display. Through garden landscaping techniques, The display of live medicinal plants is arranged in each functional area according to their different growth characteristics and functions, to express the beauty of the whole Chinese herbal garden. Scientific exhibitions of medicinal plants are presented in text and pictures as well as multimedia and small pharmaceutical workshops. The "Four Great Southern Medicines" such as betel nut, puzzle nut, sand nut, Morinda Officinalis, "Ten Great Southern Medicines" - Morinda Officinalis, Gouliang ginger, money white flower snake, sand nut, Buddha's hand, Guang Chen Pi, incense, Guang Huo Xiang, Lingnan minority medicine Miao Miao medicine cinnabar root, potato buttercups, caraway, octagonal maple, wood butterfly, abacus, etc., "Wang Lao Ji" classic medicinal plants Chrysanthemum, honeysuckle, egg flower, licorice, Xian Cao, Xia Ku Cao Cao, ragweed, ragweed, etc. are displayed intensively to facilitate the comparison and memory of visitors and learners, to achieve benefits in science exhibition and landscape viewing effect ${ }^{[4-8]}$.

\section{Status of the Medicinal Plant Garden}

The inherent deficiencies in the planning and design of the medicinal plant gardens have led to the loss of the original value of the garden, which was built less than a few years ago.

The medicinal plant garden is atypical gardens, covering all botanical taxonomic families and genera, and the planning of the medicinal plant gardens has high requirements for designers. Most of the medicinal plant gardens are invested or led by the government, and the design units must be determined by open tender, the scientific research units which are capable of planning the medicinal plant gardens are generally unable to conduct the project because of qualifications and other reasons. The project is normally handled by the company which has the forest and garden landscaping architecture qualifications. These companies use a low budget in a reduced amount of research units in the listing of plants in construction of medicinal plant gardens. Therefore, a lot of mistakes were made in the listing of medicinal plants in the medicinal plant gardens as there were misunderstanding in the name list based on "China Plan Magazine". Besides, the botanical expertise of the design unit is not enough to make up the list of medicinal plants. The botanical expertise of the construction unit is not enough to control the gate, the construction unit is also according to the construction plans, without any professional skills ${ }^{[9]}$.

Medicinal plant garden projects generally stipulate that medicinal plants to be planted within a specific number of months, which is against the natural growth of medicinal plants. The best duration for a medicinal plant garden planting is one complete life cycle of a medicinal plant, including spring, summer, autumn and winter. With spring sowing and autumn harvesting, the planting time should follow the growth habit of the plant.

There is a lack of subsequent ongoing maintenance of the medicinal plant garden and difficulty in the building of the medicinal plant garden project. Nevertheless, the most difficult issue is the maintenance of medicinal plants after the completion and hand over of medicinal plant garden to the construction units. For any plants in the construction of the garden, the landscape is also about three parts of planting and seven parts of maintenance. If the follow-up management cannot be kept up, the medicinal plant garden project will be becoming less attractive. This is a common problem of gardens in China, which involves the lack of maintenance expertise and awareness, and much traditional Chinese medicine in the gardens have operational difficulties or high mortality rates and have lost their original value.

\section{If medicinal plant cultivation techniques are strengthened}

\subsection{Techniques for the introduction and domestication of medicinal plants}

Adhere to the use of mainly native varieties, plant introduction and domestication refers to artificial cultivation, natural selection and artificial selection, so that wild plants and exotic plants can adapt to the local natural environment and planting conditions, and become the production or ornamental needs of native plants $^{[10]}$.

Many medicinal plants are native to different regions, and the geographic variability of the regions makes the living environment and climatic requirements of medicinal plants different. Medicinal plants that are brought to another region or environment in their native habitat need to be domesticated for a certain period, and then planted in a different location when they are healthy and stable, and the survival rate of simple cultivation of exotic plants is not high. 
Progressive migration is key to the success of medicinal plant introduction to seedlings. Targeted cultivation of plants is often not accomplished in a short period of one or two generations but requires multiple locations and generations to achieve the successful cultivation. The introduced medicinal plants are domesticated for 12 to 24 months, to be gradually adapted to the local climate before planting out for the nursery. After the domestication of the seedlings, the survival of these plants will increase.

\subsection{The species number is the basis for the continuation of biological populations}

The deepening in the knowledge in plant species and planting knowledge among landscape designers and farmers ensure that the number of introduced seedlings of medicinal plants in the medicinal plant garden should preferably meet the number of biological Lingnan medicinal plants with self-propagation ability, which is the idealized concept.

\subsection{Ongoing annual replenishment of medicinal plant seedlings is fundamental to the success of medicinal gardens, as improper maintenance causes many herbs to die each year}

Since there are so many medicinal plants have very different native habitats, we need to ensure them to grow healthily as we brought them to a specific area Medicinal plant gardens are a great test to growing skills. A medicinal plant garden is only complete if the annual replanting of unviable medicinal plants is continuous and reflects a more beautiful landscape ${ }^{[11-12]}$.

\subsection{Strengthening daily maintenance is the basis for the perpetual survival of medicinal plants}

The biggest threat to medicinal plants in the garden is weeds. Some medicinal plants need more stringent requirements for the growing environment, mainly in different plants for soil texture, $\mathrm{pH}$, aeration, waterretention rate, nutrient content and other soil index requirements. Therefore, most of the herbal plants in the planting process cannot be directly planted on the ground. In terms of protection, ground cloth, gating, fixed fences (bricks, concrete strips), etc. can be used to prevent weeds growing and ensure the sustainable healthy growth of medicinal plants ${ }^{[13-14]}$.

\section{Herbal garden concept}

There are many Chinese classics and records on Chinese herbal medicine, such as Li Shizhen's "Compendium of Materia Medica", Zhang Zhongjing's "Treatise on Cold and Injury" and so on.

We cannot live without Chinese herbs, but how to let people know more about Chinese herbs and discover their medicinal value is an issue that we should ponder. Therefore, is it important in applying the Chinese medicinal plants in the garden landscaping and science teaching materials in school, and healthy medicinal gardens.

\section{References}

[1] Liu CX, Wang YL, Zhang HB, et al.Based on the novel Coronavirus infection prevention and control requirement, it attaches importance to the scientific research and application of Chinese medicine [J].Chinese herbal medicine, 2020, 51(6): 1361-1374.

[2] Ni LQ, Tao HW, Yang XL, et al. (in Chinese)Chinese journal of traditional Chinese medicine,2020,38(4): 8-14.

[3] Xia WG, An CQ, Zheng QJ, et al.Chinese journal of traditional Chinese medicine, 2020, 61(5): 375-382.

[4] Xu X, Zhang Y, Li X, et al.Analysis of TCM prevention of COVID-19(COVID-19) programs in different regions [J]. Chinese herbal medicine, 2020, 51(4): 866-872.

[5] Li LW, Xu XF, Yu XH.Application of medicinal plants in landscaping [J]. Modern Horticulture, 2019(12): 145.

[6] Chen C. Discussion on the planning and design of special gardens for medicinal plants based on the theme of Traditional Chinese medicine culture [D].Southwest University,2015.

[7] Hao Y. Research on Landscape Participatory Design of Botanical Garden [D]. Fujian Agriculture and Forestry University, 2014.

[8] Li S. Expression of Chinese Medicine Culture in Landscape Design [D]. Southwest University,2013.

[9] Wang YJ. Exploration of Medicinal plants and Their Landscape in Guangxi [D]. South China University of Technology, 2010.

[10] Luo D R. Preliminary Study on the Planning and Design of medicinal Plant Gardens [D]. Southwest University, 2009 (in Chinese)

[11] Ding HJ, Zhang YK.Niche Design and plant configuration of Baicao Garden, Hangzhou Botanical Garden [J]. Huazhong Architecture,2007(10):133-136.

[12] Meng XH. Key points of planning and Design of Special Gardens for Medicinal Plants [J]. Anhui Agricultural Science, 2007(21):6461+6550.

[13] Zeng Y. Research on the Development Model of Hainan Ecological Pharmaceutical Industry[D]. Shanghai Jiao Tong University, 2006.

[14] Mao YZ, Chen W. Planning and Design of medical Botanical Gardens attached to schools[J]. Health Vocational Education, 2003(12): 24-25. 